\title{
ARTICLE
}

\section{Source Reconstruction of Unknown Model Parameters in Atmospheric Dispersion Using Dynamic Bayesian Inference}

\author{
Joo Yeon $\mathrm{KIM}^{1 *}$, Han-Ki JANG ${ }^{1}$, and Jai Ki LEE ${ }^{1}$ \\ ${ }^{1}$ Hanyang University, 17, Haengdang, Seongdong,Seoul 133-791, Korea
}

\begin{abstract}
A source reconstruction capability driven by time-evolving data is tried out by coupling the observed data and predictive model through dynamic Bayesian inference to obtain solutions to inverse problem. Solutions are determined by posterior probability distributions describing unknown model parameters and the Markov Chain Monte Carlo method with the Metropolis-Hastings sampling algorithm is employed to obtain the solutions. The posterior distributions of model parameters are obtained by performing stochastic sampling by the likelihood function test indicating the agreements between the measurements and the predictions from Gaussian plume model. The Yonggwang atmospheric tracer experiment in Korea is selected to testify the source reconstruction algorithm. The simulation has shown that the posterior modes of the release point and the released source rate for this experiment obviously converged to their true values.
\end{abstract}

KEYWORDS: atmospheric dispersion, source reconstruction, dynamic Bayesian inference, Markov Chain Monte Carlo, Metropolis-Hastings sampling algorithm

\section{Introduction}

Atmospheric releases of hazardous material due to an accident at nuclear facility or a radiological attack can affect large populations, and cause significant damage to human health and the environment. Reliable predictions of air concentration are extremely valuable in guiding an effective and timely response.

However, all of the model parameters are not necessarily known well in certain cases particularly in emergency situations where severe time constraints are imposed. Instead, the results of dispersion, for examples air concentrations at certain locations on the site of interest, may be available from a monitoring network. A probabilistic source reconstruction model that couples field measurements with dispersion model predictions can be applied in order to evaluate the unknown model parameters characterizing the state of event. This process constitutes a backward model or an inverse model.

In this study, inverse tracking of unknown model parameters characterizing atmospheric dispersion event has been tried out by coupling a time-dependent data stream with a predictive model in a manner that allows dynamic improvement in estimates of the parameters as the measurements become available. The inverse algorithm is based on dynamic Bayesian inference combined with a Markov Chain Monte Carlo (MCMC) method using the Metropolis-Hastings (M-H) sampling algorithm. ${ }^{1)}$ The Yonggwang (YG) atmospheric tracer experiment performed in Korea is selected for the source reconstruction ${ }^{2)}$ and unknown model parameters are assumed as the release point $(x, y)$ and the released source rate $(Q)$.

\section{Materials and Method}

\section{Applying Bayes' Theorem to Source Reconstruction}

Bayes' theorem defines the posterior probability of a set of model parameters $\boldsymbol{\theta}$ given the measurements $\boldsymbol{d}$ from a sensor network.

$$
p(\boldsymbol{\theta} / \boldsymbol{d}) \propto p(\boldsymbol{\theta}) L(\boldsymbol{d} / \boldsymbol{\theta})
$$

$p(\boldsymbol{\theta} / \boldsymbol{d})$ is the posterior distribution. $p(\boldsymbol{\theta})$ is the prior distribution and represents prior knowledge about the model parameters before obtaining the data. In this study, non-informative or uniform distribution is applied as prior ones for three model parameters. $L(\boldsymbol{d} / \boldsymbol{\theta})$ is the likelihood function and models the agreements between the measurements and the predictions. The consideration for constructing the likelihood function is to reflect zero observations below its threshold value within the function framework. Therefore, the likelihood function accounting for zero sensor readings is written as Eq. (2).

$$
\begin{aligned}
& L(\boldsymbol{d} / \boldsymbol{\theta})=I[d=0] \exp \left(-\alpha C_{m}\right) \\
&+I[d>0] \frac{1-\exp \left(-\alpha C_{m}\right)}{\sqrt{2 \pi} \sigma d} \exp \left(-\frac{\left(\ln d-\ln C_{m}\right)^{2}}{2 \sigma^{2}}\right)
\end{aligned}
$$

For a further review of this likelihood function, refer the following literature. ${ }^{3)}$

A simple Gaussian plume model is applied as the forward one to predict $C_{m}$ and this model for uniform steady wind conditions can be written as 


$$
\begin{aligned}
C_{m}= & \frac{Q}{2 \pi U \sigma_{y} \sigma_{z}} \exp \left(-\frac{y^{2}}{2 \sigma_{y}^{2}}\right) \\
& \left\{\exp \left(-\frac{(z-H)^{2}}{2 \sigma_{z}^{2}}\right)+\exp \left(-\frac{(z+H)^{2}}{2 \sigma_{z}^{2}}\right)\right\}
\end{aligned}
$$

where $Q(\mathrm{~g} / \mathrm{s})$ is the released source rate, $U(\mathrm{~m} / \mathrm{s})$ is the mean wind speed, $H(\mathrm{~m})$ is the release height, $x$ is the distance along the wind, $y$ is the distance along the horizontal crosswind direction and $z$ is the distance along the vertical axis defining the sensor heights. $\sigma_{y}$ and $\sigma_{z}$ are called as the standard deviations in the horizontal crosswind and vertical direction, respectively. These two standard deviations are defined empirically for different stability conditions and are presented as Eq. (4).

$$
\sigma_{i}=a_{i} x\left(1+b_{i} x\right)^{-c_{i}}
$$

The values of the constants $a_{i}, b_{i}$ and $c_{i}$ have been given by Brodsky. ${ }^{4)}$

The approximate solutions of Eq. (1) can be obtained by MCMC simulation based on the M-H sampling algorithm. In this algorithm, a candidate state $\phi$ is sampled from a proposal distribution with probability of Eq. (5) at each iteration

$$
\alpha(\boldsymbol{\theta}, \boldsymbol{\phi})=\operatorname{Min}\left\{1, \prod_{n=1}^{N} \frac{\pi(\boldsymbol{\phi})}{\pi(\boldsymbol{\theta})}\right\}
$$

where, $N$ is the total number of sensors and $\boldsymbol{\theta}$ is the current states of the interesting model parameters. For a further review of this algorithm, refer the following literature. ${ }^{5,6)}$

For generating Markov chains, two sets of initial values are assumed as $(5,3,22)$ and $(1,-3,20)$, where they correspond to $(x, y, Q)$. The simulations of 5000 by each time-evolving stage are applied for source reconstruction and total simulations are then to be 15000 . Additionally, the first half simulations 7500 are assumed as the burn-in for deriving the more reliable target distributions for interesting model parameters and they are discarded for the source reconstruction.

\section{Yonggwang Atmospheric Tracer Experiment}

The part of the measurements, which has been obtained through the experiments performed in the YG nuclear power reactor site of Korea in May 29, 1996, is used for the source reconstruction. The weather conditions have been measured in five points around nuclear power reactor, and data of wind direction, stability class and wind speed determined as 48 consecutive ten-min averaged values. The stability class is to be $\mathrm{B}$, and the wind direction and the wind speed are to be $242 \mathrm{deg}$ and $4.9 \mathrm{~m} / \mathrm{s}$, respectively. The wind direction means to increase toward clockwise direction on the north of the

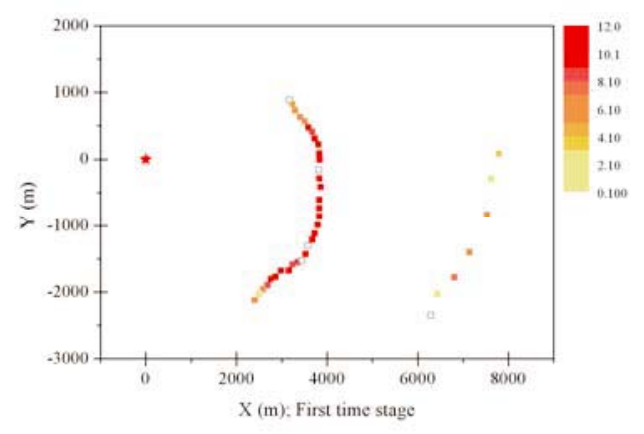

(a) First time stage

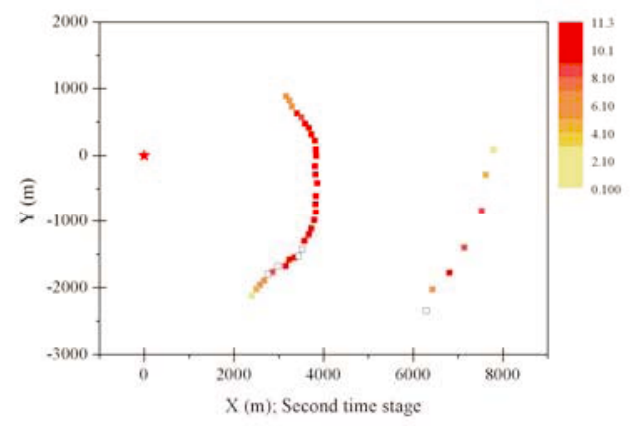

(b) Second time stage

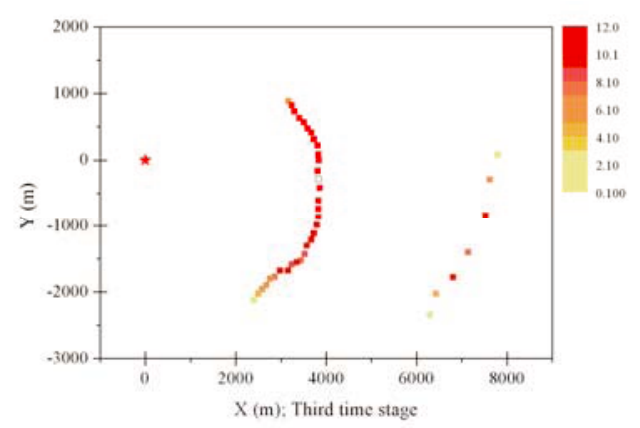

(c) Third time stage

Fig. 1 Yonggwang atmospheric tracer experiment. Clear indications and a star one represent zero concentrations and true release point, respectively.

release point. The release point and the release height are to be the origin and $58 \mathrm{~m}$, respectively, and $\mathrm{SF}_{6}$ gas has been released as the constant rate of $32 \mathrm{~g} / \mathrm{s}$ from 15:00 to $16: 30$. For measuring tracer concentrations, 91 sensors have been located in 2 to $8 \mathrm{~km}$ from the release point, and six 10-min averaged concentrations recorded. In this study, the first three 10-min averaged measurements are used for source reconstruction as summarized in Fig. 1.

\section{Results and Discussion}

\section{Posterior Distributions}

A program for source reconstruction is written by using $\mathrm{C}^{++}$language. According to dynamic Bayesian inference, note that simulations should be consecutively performed by considering the data measured in each time stage. That is, the first source reconstruction is performed by applying the first measurements, and the second source reconstruction is then performed by applying the second measurements and the posterior results obtained from the first source reconstruction, 
and so on.

The bimodal posterior distribution for the release point has been depicted by each time-evolving stage in Fig. 2. Clear circles mean the true values of the release point. The ranges of $x$ and $y$ axes of the release point are decreased with approach to the third time-evolving stage, and their uncertainties have been then considerably removed through dynamic Bayesian inference. The true value of the release point satisfies within $11 \%$ ile contour line of the histograms in detail and has approximated to the bimodal posterior modes regardless of time-evolving stage.

The posterior distribution for the released source rate has been depicted in Fig. 3 as well. The range and uncertainty of

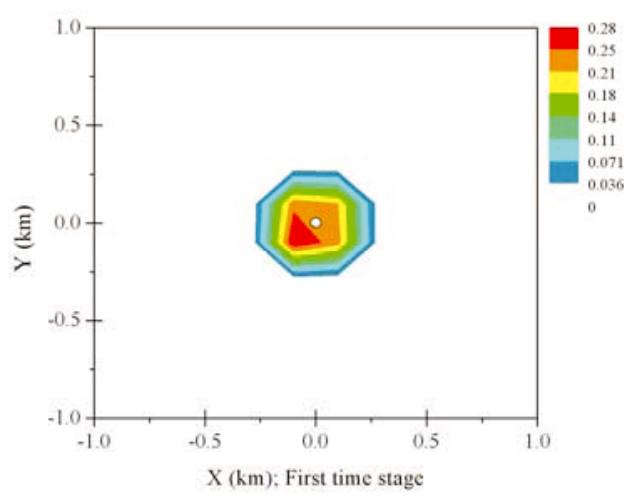

(a) First time-evolving stage

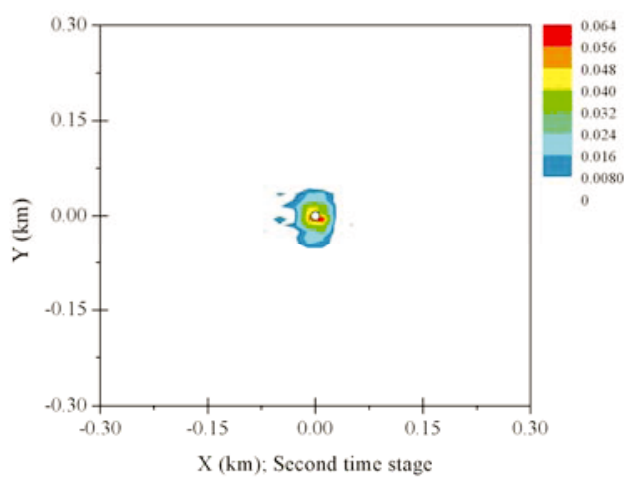

(b) Second time-evolving stage

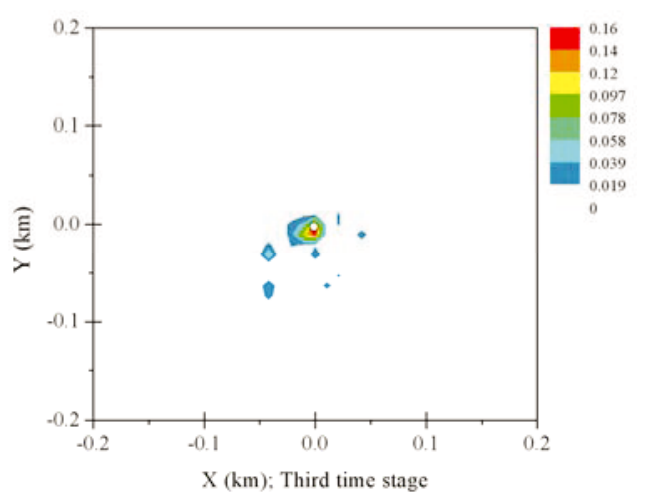

(c) Third time-evolving stage

Fig. 2 Bimodal posterior distributions of the release point the posterior distributions are decreased with approach to the third time-evolving stage. Its posterior modes are obviously accorded with $32 \mathrm{~g} / \mathrm{s}$ for all time-evolving stages, and it is then possible to infer the true value of the released source rate. The unknown model parameters in atmospheric dispersion have been successfully tracked through source reconstruction tried out in this study.

However, $\sigma$ defining the agreements between the measurements and the predictions in Eq. (2) has been turned out to be about 500 to $900 \%$. There are several reasons for this mismatch between the predictions and the measurements for the source reconstruction in YG tracer experiment. First of all, the major reason for the mismatch between the two concentrations is that all measured concentrations are averaged values from a 10 -min release, whereas model predictions are steady-state results. And, the differences

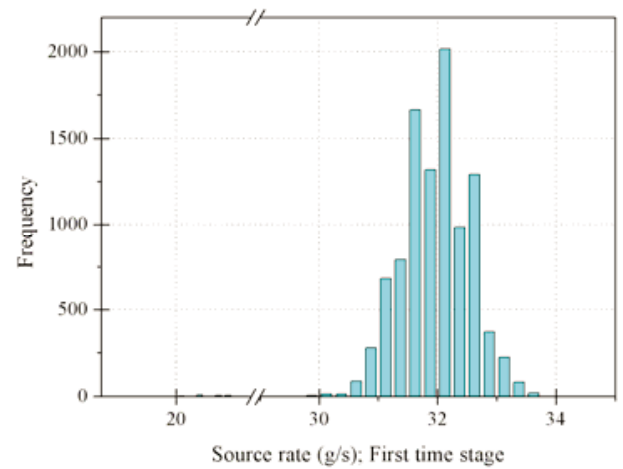

(a) First time-evolving stage

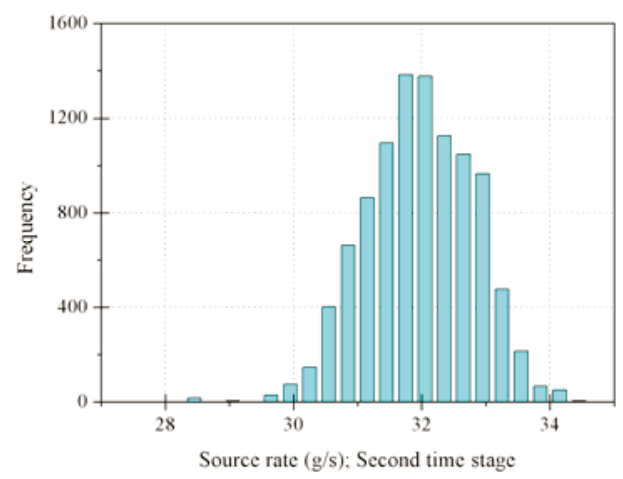

(b) Second time-evolving stage

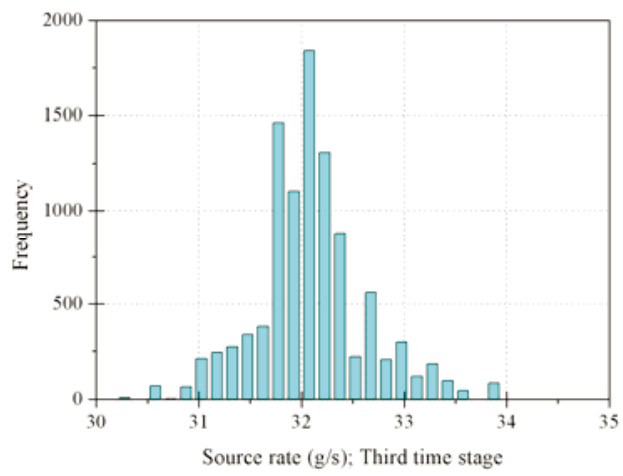

(c) Third time-evolving stage

Fig. 3 Posterior distributions of the released source rate 


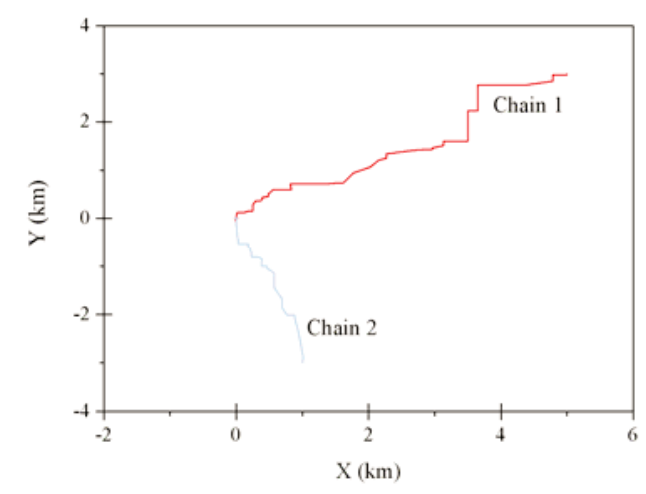

Fig. 4 Posterior trajectory of the release point

between the measurement and the predictions are not constant, because the measurements at the same sensor locations have been much varied by time stage.

From these reasons, for inverse tracking unknown model parameters in the source reconstruction turned out to be remarkable differences of the measurements at same sensor locations, it may be preferably advantageous that only one source reconstruction is performed by applying the agreements between the average measurements for all time stages and the predictions without considering dynamic Bayesian inference.

\section{Posterior Trajectory for the released point}

The most important model parameter in atmospheric dispersion event may be the release point. The posterior trajectories contribute, therefore, to examining whether the true release point can be efficiently tracked independent of some starting points or not. Fig. 4 shows the posterior trajectories for the release points of the two Markov chains, and ensures that the release point clearly indicates the true value of $(0,0) \mathrm{km}$ independent of starting points.

Through examining these posterior distributions and the posterior trajectory of the release point, unknown model parameters have enough achieved their target distributions. The inverse tracking algorithm tried out in this study has been successful in reconstruction of unknown or uncertain source parameters. These results can contribute to a decision-making drawn for an emergency response under severe atmospheric dispersion conditions.

\section{Conclusion}

A probabilistic inference for inverse tracking of model parameters for atmospheric dispersion, a set of measurement data is given, has been presented for application to characterization of the event. It combines dynamic Bayesian inference with Markov Chain Monte Carlo method based on the Metropolis-Hastings sampling algorithm. The probabilistic simulations have shown that the posterior distributions of the model parameters achieve their target distributions, and the source reconstruction is then successful by indicating the true release point within the bimodal posterior distributions. The posterior mode of the released source rate has also indicated the true value $32 \mathrm{~g} / \mathrm{s}$. The uncertainty of posterior distribution has been successfully decreased to an insignificant level through dynamic Bayesian inference by three time stage.

The probabilistic aspect of the solution optimally combines a likely answer with the uncertainties of the available data. Among several possible solutions, the Bayesian source reconstruction is solely able to find values of the model parameters that are more consistent with the data available and its uncertainties. The source reconstruction performed in this study would provide decision-makers with better information about the accident situations, soon after the first measurements were available. This would lead to timely and efficient response actions against the emergency situations.

\section{Acknowledgment}

This work was supported by Korean Ministry of Knowledge Economy (2008-P-EP-HM-E-06-0000) and Sunkwang Atomic Energy Safety Co., Ltd..

\section{References}

1) D. Gamerman, Markov Chain Monte Carlo: Stochastic Simulation for Bayesian Inference, 2nd ed., Chapman \& Hall/CRC, Boca Raton (2002).

2) M. H. Han, E. H. Kim, K. S. Suh, W. T. Hwang, H. J. Jeong, Development of Long-Range Radiological Emergency Preparedness Technology, KAERI/RR-2325/2002, Korea Atomic Research Intitute (Korean) (2002).

3) I. Senocak, N. W. Hengartner, M. B. Short, W. B. Daniel, Stochastic Event Reconstruction of Atmospheric Contaminant Dispersion Using Bayesian Inference, Atmos. Environ. 42(33) (2008) 7718-7727.

4) R. E. Faw, J. K. Shultis, Radiological Assessment: Source and Exposure, PTR Prentice-Hall, Inc. (1993).

5) N. Metropolis, A. W. Rosenbluth, M. N. Rosenbluth, A. H. Teller, E. Teller, Equation of State Calculations by Fast Computing Machines, J. Chem. Phys. 21(6) (1953) 1087-1092.

6) W. K. Hastings, Monte Carlo Sampling Methods Using Markov Chains and Their Applications, Biometrika 57(1) (1970) 97-109. 